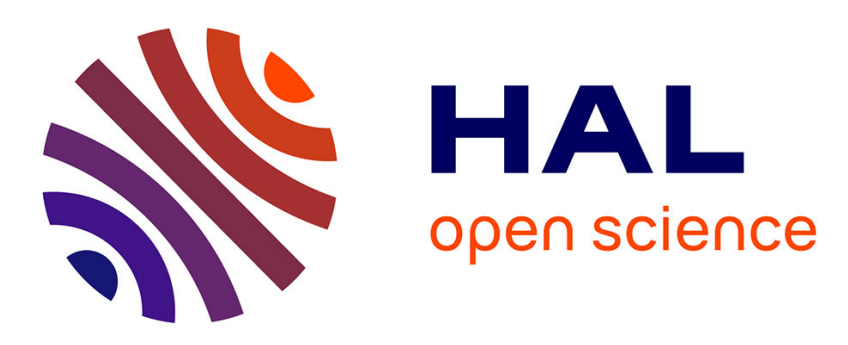

\title{
Microwave-Assisted Nitroxide-Mediated Radical Polymerization of Acrylamide in Aqueous Solution
}

Julien Rigolini, Bruno Grassl, Laurent Billon, Stephanie Reynaud, Olivier François Xavier Donard

\section{- To cite this version:}

Julien Rigolini, Bruno Grassl, Laurent Billon, Stephanie Reynaud, Olivier François Xavier Donard. Microwave-Assisted Nitroxide-Mediated Radical Polymerization of Acrylamide in Aqueous Solution. Journal of polymer science. Part A-1, Polymer chemistry, 2009, 47, pp.6919-6931. 10.1002/pola.23731 . hal-00469605

\section{HAL Id: hal-00469605 https://hal.science/hal-00469605}

Submitted on 1 Apr 2010

HAL is a multi-disciplinary open access archive for the deposit and dissemination of scientific research documents, whether they are published or not. The documents may come from teaching and research institutions in France or abroad, or from public or private research centers.
L'archive ouverte pluridisciplinaire HAL, est destinée au dépôt et à la diffusion de documents scientifiques de niveau recherche, publiés ou non, émanant des établissements d'enseignement et de recherche français ou étrangers, des laboratoires publics ou privés. 


\title{
Microwave-Assisted Nitroxide-Mediated Radical Polymerization of Acrylamide in Aqueous Solution
}

Julien Rigolini, Bruno Grassl *, Laurent Billon, Stephanie Reynaud and Olivier F.X. Donard Université de Pau et des Pays de l'Adour, IPREM UMR CNRS/UPPA 5254, Hélioparc Pau Pyrénées - 2 Avenue du Président Angot - 64053 Pau cedex 09, France

* Corresponding author (e-mail: bruno.grass1@univ-pau.fr)

\begin{abstract}
:
The present work describes a combination of microwave irradiation as a heating source and water as a solvent for carrying out a living/controlled polymerization of acrylamide. Reasonable results were obtained for a nitroxide-mediated radical polymerization (NMP) with a combination of a conventional hydrosoluble radical initiator and a $\beta$-phosphonylated nitroxide. The microwave enhancement of the polymerization was found to depend on the mode of irradiation, i.e., either a DYN mode or an SPS mode. The former mode corresponded to a dynamic control of the temperature by way of a high initial microwave power, and in this case, no specific microwave effect was observed. On the other hand, in the SPS mode, which is a pulsed power mode, the result showed a strong acceleration of the polymerization process (> 50 times) without the loss of the living/controlled polymerization characteristics which is relevant with a re-initiation of the polyacrylamide macroinitiator even after $100 \%$ of conversion.
\end{abstract}

Keywords: microwave, acrylamide, controlled/living polymerization, Nitroxide Mediated Polymerization. 


\section{Introduction}

Controlled radical polymerization (CRP) is a well-known route for preparing (co)polymers with controlled functionalities and topologies, as well as targeted molar masses with low polydispersities. The CRP process requires selecting conditions that allow, or create, a dynamic equilibrium between a low concentration of active propagating chains and a large number of dormant chains, which are unable to propagate or self-terminate. There exist several CRP processes based on this fundamental understanding and the techniques that have received the most attention include Atom Transfer Radical Polymerization, (ATRP); Stable Free Radical Polymerization (SFRP), e.g., Nitroxide-Mediated Polymerization (NMP) and OrganoMetallic Radical Polymerization (OMRP); Degenerative Transfer (DT) processes, e.g., Reversible Addition Fragmentation Transfer (RAFT), and Macromolecular Design via the Interchange of Xanthates (MADIX). ${ }^{1}$

Microwave-assisted polymer chemistry is a rapidly growing field of research. In the last few years, various examples of reaction accelerations, selectivities, and higher yields have been reported. ${ }^{2-9}$ Overviews on microwave-assisted polymerizations have recently been provided by Ritter ${ }^{10,11}$ as well as Schubert and co-workers, ${ }^{12-14}$ whereby special attention was given to microwave-assisted CRP. In resumé, the polymerization rates were found to be clearly faster under microwave (MW) irradiation than under classical thermal heating at identical polymerization temperatures and under controlled conditions. This was true for NMP of styrene in bulk ${ }^{15}$, and methyl acrylate and tert-butyl acrylate in a relatively polar solvent $(1,4-$ dioxane); ${ }^{16}$ for RAFT polymerization of methyl methacrylate, ${ }^{17}$ vinyl acetate, ${ }^{17}$ and styrene, ${ }^{17,18}$ and for ATRP of methyl methacrylate ${ }^{19-21}$ and acrylonitrile in refluxing tetrachloromethane. ${ }^{22}$ The effectiveness was seen to depend essentially on the method used for performing the MW-assisted polymerization. Even faster polymerization rates have mostly been obtained under MW irradiation. It is indeed difficult to compare the results due to the great variety of experimental conditions described within the studies. Results have been obtained with a domestic or monomode MW oven and various modes of irradiation. Demonceau $^{19}$ showed that a microwave effect was noted during ATRP of methyl methacrylate; an effect that strikingly depended on both the temperature and the method used, e.g., conventional microwave synthesis (CMS) or enhanced microwave synthesis (EMS), in the monomode MW oven. Their results differed from those reported by Schubert and coworkers ${ }^{20}$ for the microwave-assisted ATRP of MMA using a copper-based system, thereby 
demonstrating the absence of any 'microwave effect' in ATRP, in contrast to several literature reports ${ }^{19,21}$, but without a consistent comparison of the irradiation method in terms of power and energy supplied to the reaction medium.

Microwave effects are obviously the subject of considerable current debate and controversy, and extensive research efforts are necessary to truly understand these and related phenomena. On the other hand, the combined use of microwave irradiation as a heating source and water as a solvent for synthetic organic transformations is a relatively new approach, as recently reported by Kappe et $a .^{23}$ Nevertheless, to the best of our knowledge, work involving microwave-assisted CRP in aqueous media has yet to be published. Recently, Erdmenger et al. have used water as solvent for the microwave-assisted free radical polymerization of styrene ${ }^{24}$.

Thus, the issue of microwave effects on the controlled radical polymerization in water is the primary focus of this work. For that, the choice was made to carry our microwave-assisted NMP of acrylamide at high concentrations (40 wt-\%) in a purely aqueous solution, using a combination of a conventional hydrosoluble radical initiator (Vazo56) and a $\beta$ phosphonylated nitroxide, SG1. Evidence of a living/controlled nature of the polymer is presented and the effects of the employed MW irradiation method are described.

Unlike other polyacrylamide derivatives that are soluble in common organic solvents, poly(acrylamide) PAM is only soluble in water, dimethylsulfoxide and glycerol. Moreover, the monomer is a powder, which inhibits the bulk polymerization from being considered at a reasonable temperature. Under such constraints, the controlled radical polymerization, CRP, of acrylamide in aqueous solution seems to be a realistic route, as both monomer and polymer are highly soluble in water up to $40 \mathrm{wt} \%$, and respect the environmental benefits associated with microwave-assisted aqueous polymerizations. ${ }^{25,26}$

\section{Experimental section}

\subsection{Materials}

Acrylamide (AM) (purity >99\%) was purchased from ABCR, and 2,2-azobis (2methylpropionamidine) dihydrochloride, denoted VAZO56, was obtained from Dupont Chemicals. N-tert-butyl- N-[1-diethylphosphono-(2,2-dimethylpropyl) nitroxide] (DEPN), also called SG1, was kindly provided by Arkema and used as the counter radical. All 
chemical products were used as received. Deionized water, with an electrical conductivity of 18.3 M $\Omega . \mathrm{cm}$ at $25^{\circ} \mathrm{C}$, was filtered through a $0.22 \mu \mathrm{m}$ Millipore filter prior to use.

\subsection{Polymerization}

A bimolecular system, based on Vazo56 (initiator) and free SG1 (counter radical) was used to run polymerizations of $\mathrm{AM}$ at $40 \mathrm{wt} \%$. The molar ratio $[\mathrm{SG} 1]_{0} /[\mathrm{Vazo} 56]_{0}$ was kept constant at 1.8 according to previous optimizations carried out for acrylamide ${ }^{27}$ and organosoluble monomer. $^{28,29}$ Monomer solutions, containing a constant amount of initiator $\left([\mathrm{AM}]_{0} / 2[\text { Vazo56 }]_{0}=500\right)$ were purged with nitrogen for 5 min directly in a $15-\mathrm{mL}$ Pyrex culture tube with a screw-cap, or in a standard 10-mL Pyrex microwave process vial before being heated in an oil bath or through microwave irradiation. A total amount of $4.5 \mathrm{~mL}$ and 3 $\mathrm{mL}$ were heated conventionally in an oil bath and by way of microwave experiments, respectively. The polymerization was terminated by cooling in an ice bath (for the case with conventional heating in an oil bath) or with compressed air (for the microwave experiments) and the reactive medium was sampled for size exclusion chromatography coupled with light scattering detector (SEC-MALS). For the kinetic experiments, the same reaction mixture has been used to prepare several tubes and each tube was withdrawn at different time intervals.

\subsection{Size exclusion chromatography coupled with multi-angle light scattering (SEC-MALS)}

The number-average molar mass $\left(\mathrm{M}_{\mathrm{n}}\right)$, polydispersity (Ip), and polymer conversion (p) of the polyacrylamide (PAM) samples were determined by size exclusion chromatography (SEC) using a Waters Alliance 2690 (USA) chromatograph equipped with two Shodex OHpak columns (SB-804HQ, and SB-802.5HQ) and two online detectors: a differential refractometer and a DAWN ${ }^{\circledR}$ HELEOS $^{\mathrm{TM}}$ II utilizing a $120-\mathrm{mW}$ solid-state laser operating at $658 \mathrm{~nm}$ and fitted with a $\mathrm{K} 5$ cell. A $0.1-\mathrm{M}$ solution of $\mathrm{NaNO}_{3}$ was used as the eluent at a flow rate of 0.5 $\mathrm{mL} / \mathrm{min}$. Samples were prepared by diluting approximately 20 times the reaction mixture in $0.1 \mathrm{M} \mathrm{NaNO}_{3}$ and then filtering with a $0.45 \mu \mathrm{m}$ filter (Millipore) after about 1 hour of stirring. Samples of 25 to $75 \mu \mathrm{L}$ of these solutions were injected. The number-average molar mass and polydispersity were obtained from data collected and analyzed using the ASTRA SECsoftware (version 5.3, Wyatt Technology Corp., USA). The calculation of molar mass was carried out according to the Zimm fit method. The differential molar mass distribution was carried out according to the common form: $x(M)=d W(M) / d \log (M)$, where $W(M)$ is the cumulative distribution (the weight fraction of sample having molar mass less than $\mathrm{M}$ ). The refractive index increment, $\mathrm{dn} / \mathrm{dc}$, was determined to be respectively $0.184 \pm 0.001$ and 
$0.144 \pm 0.001 \mathrm{~mL} / \mathrm{g}$ for polyacrylamide and acrylamide according to the method described in the literature. ${ }^{30,31}$

Figure 1: SEC-MALS results for a typical experiment: $\mathrm{CH}$ run, $120^{\circ} \mathrm{C}, \mathrm{t}=20,40$ and $50 \mathrm{~min}$ (see Table 1). a) Chromatograms (top), and b) molar mass distribution (bottom). The three peaks on the left of the top figure correspond to the three peaks in the bottom figure.
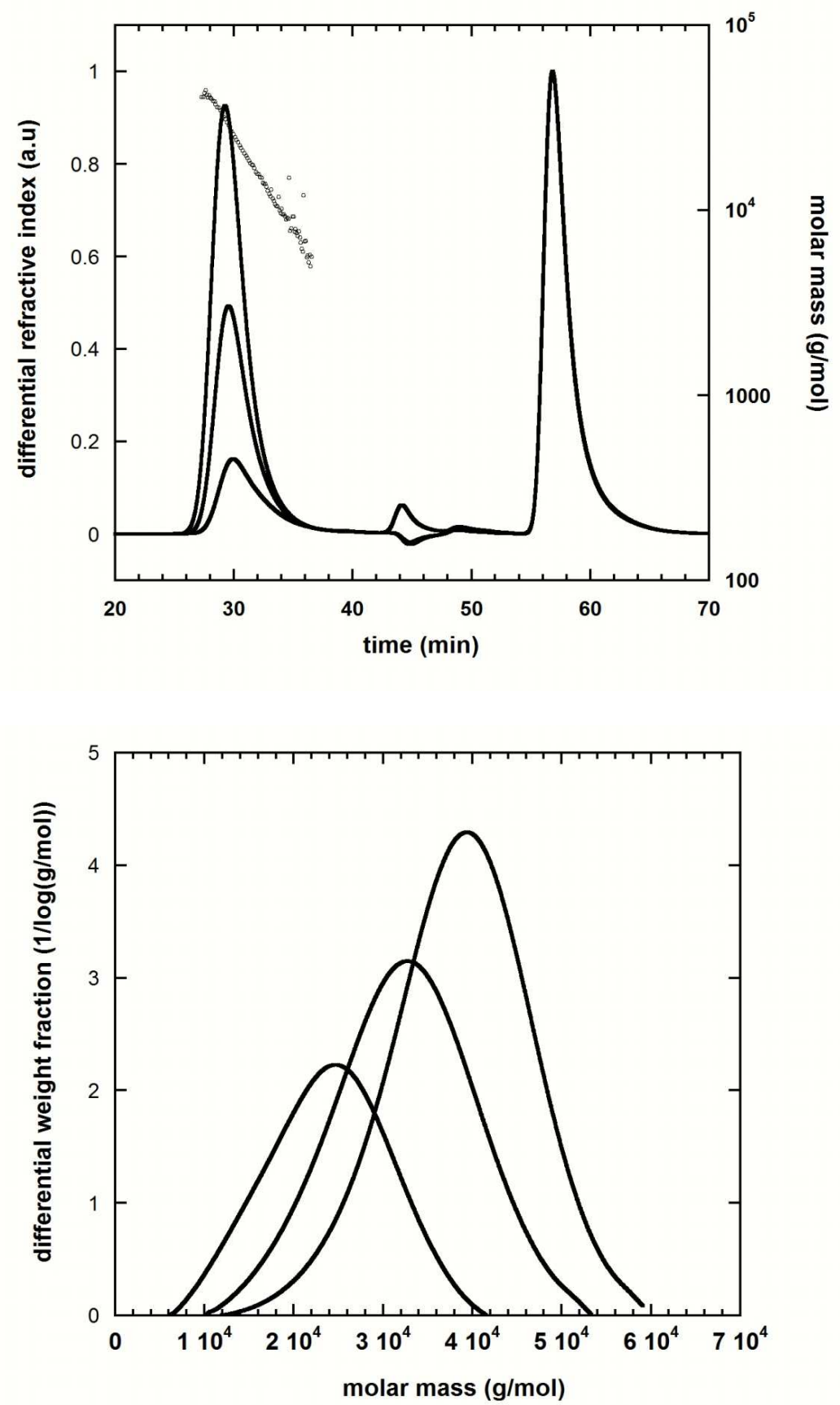

Figure 1 present a typical trace from SEC experiments on reactive media, displaying two characteristic peaks of the PAM polymer and acrylamide (AM) monomer. With knowledge of the refractive index increment of acrylamide and polyacrylamide in $0.1 \mathrm{~mol} . \mathrm{L}^{-1} \mathrm{NaNO}_{3}$ 
solution, the polymer conversion, $\mathrm{p}$, could be computed from the surface areas of the PAM and AM peaks, respectively:

$$
\mathrm{p}=\mathrm{S}_{\mathrm{PAM}} /(\mathrm{dn} / \mathrm{dc})_{\mathrm{PAM}} /\left(\mathrm{S}_{\mathrm{PAM}} /(\mathrm{dn} / \mathrm{dc})_{\mathrm{PAM}}+\mathrm{S}_{\mathrm{AM}} /(\mathrm{dn} / \mathrm{dc})_{\mathrm{AM}}\right)
$$

Here, $S_{\mathrm{PAM}}, \mathrm{S}_{\mathrm{AM}}$ and $(\mathrm{dn} / \mathrm{dc})_{\mathrm{PAM}},(\mathrm{dn} / \mathrm{dc})_{\mathrm{AM}}$ are respectively the surface area and the refractive index increment of PAM and AM.

The coupling of SEC with multi-detectors, such as a refractometer (RI) or a multi-angle light scattering detector, was performed in order to determine both the polymer conversion and the molar masses of PAM with decent accuracy. It is worth noting that systematic errors occurred due to the incorrect values of $\mathrm{dn} / \mathrm{dc}$ and to the refractometer calibration factor, $\mathrm{k}$. The effects of RI calibration on the polymer conversion and molar mass were found to be proportional to $\mathrm{k}^{-1}$; the effects of $\mathrm{dn} / \mathrm{dc}$ on $\mathrm{p}$ and Mw were respectively proportional to $\mathrm{dn} / \mathrm{dc}$ and $(\mathrm{dn} / \mathrm{dc})^{-1}$. For the $\mathrm{p}$ and $\mathrm{Mw}$ values, these systematic errors could be prevented by using the same refractometer detector for determining $\mathrm{dn} / \mathrm{dc}$ and $\mathrm{p}$. The error limits of $4 \%$ and $8 \%$ for $\mathrm{p}$ and Mw, respectively, were based on the averages and standard deviations from multiple experiments. The random errors inherent to each experiment were due to baseline fluctuations of each detector.

\subsection{Viscometric measurements of polymer solutions}

The steady shear rheological properties of the polymer solutions were measured with a Bohlin CVOR150 rheometer in controlled stress mode with a cone-plate geometry (radius $=20.0$ $\mathrm{mm}$, angle $=0.096 \mathrm{rad}$, gap $=0.150 \mathrm{~mm}$ ). Stepped shear stress values were selected in order to allow shear rates ranging from 0.01 to $150 \mathrm{~s}^{-1}$. The systems were allowed to reach steady state at each shear stress prior to registering the measured values. The temperature was controlled with a Peltier plate system and was set to $20.0 \pm 0.1^{\circ} \mathrm{C}$.

\subsection{Microwave Methods (DYN and SPS)}

The NMP of acrylamide in aqueous solution was performed either in a Discover single-mode microwave synthesizer (from CEM Corp.) using the DYN and SPS modes, or in an oil bath with conventional heating, denoted $\mathrm{CH}$. The DYN mode corresponds to a dynamic control of the temperature by use of a high initial microwave power, and the SPS mode is a pulsed power mode. In each case, the volume ratio between the reaction mixture and the reactor was maintained unchanged to provide a constant pressure during the polymerization process. Microwave-assisted polymerizations were carried out in standard Pyrex vessels (total capacity $10 \mathrm{~mL}$ ) sealed with a Teflon septum cap. The temperature profiles of the polymerization 
(power control) were monitored using a calibrated infrared temperature control mounted beneath the reaction vessel. The reaction parameters (temperature, power, cooling, stirring...) were set with a laptop and the SYNERGY software (version 1.35 from CEM Corp.). Each mode of irradiation corresponded to a specific set of parameters. For the pulse mode (SPS), these parameters included the power output ( $1 \mathrm{~W}$ to $300 \mathrm{~W})$, the reaction time, the average temperature and the difference between this temperature and the lowest or highest bulk temperature (in our experiments $\pm 2^{\circ} \mathrm{C}$ ) and the maximum pressure allowed, i.e. the pressure at which the apparatus should stop the synthesis (17 bar in all experiments). The cooling system, based on a flow of compressed air at ambient temperature, and the stirring needed to be set at the beginning of the reaction (speed set to high). For the dynamic mode (DYN), the parameters were the bulk temperature, the maximum power output allowed $(300 \mathrm{~W})$, the prereaction time of stirring $(0 \mathrm{sec})$, the ramp time, i.e., the maximum time during which the apparatus was allowed to reach the desired bulk temperature $(0 \mathrm{~min})$, the reaction time, the stirring (speed set to high), the cooling system (Power Max off) and the maximum pressure allowed (17 bar in all experiments).

In the DYN mode, a dynamic control of the temperature was implemented by using a high initial microwave power, as shown in Figure 2a. The monitored temperature increased rapidly, i.e., in less than a few dozens of seconds, to the desired set point, $T_{P}$. However, upon reaching this temperature, the microwave power decreased or was shut off completely in order to maintain the target temperature without exceeding it. Compared to a non-polar solvent or monomer such as styrene, the $T_{p}$ was reached much faster: more precisely, to reach $\mathrm{T}_{\mathrm{p}}=105^{\circ} \mathrm{C}$, the required time was $35 \mathrm{~s}$ for acrylamide in a water solution and $350 \mathrm{~s}$ in styrene.

The SPS mode corresponded to a pulsed power mode in which the reaction temperature $\pm 2{ }^{\circ} \mathrm{C}$ was set, and attained by irradiation power. These experimental conditions were achieved by using a cooling feature consisting in compressed air. As shown in Figure $2 b$, in this mode, when the top limit of the targeted temperature was reached, the microwave power was shut off, the temperature decreased, and as soon as the lower instruction limit of the temperature was attained, the microwave power was reactivated. A pulsed MW power could thereby be obtained, providing an oscillation of the temperature around a set point. It is worth noticing that when the temperature instruction was $\mathrm{T} \pm 2^{\circ} \mathrm{C}$, the experimental conditions did not exceed $\mathrm{T} \pm 4^{\circ} \mathrm{C}$ (Figure $2 \mathrm{a}$ ), and this feature was mainly due to the inertia of the systems for one to three hours of irradiation, in the power range of 20-300W. On the other hand, the applied MW 
power could be perfectly controlled. In the SPS mode, the supply of microwave energy to the reaction mixture could thus be obtained by the following equation:

$$
\mathrm{E}_{\mathrm{MW}}=\mathrm{t}_{\mathrm{MW}} \cdot \mathrm{P}
$$

where $t_{\mathrm{MW}}$ is the time during which the nominal MW power, $\mathrm{P}$, is applied. This corresponds to the surface area under the power fit, as shown in Figure $2 b$ (integration is used for the DYN mode).

Figure 2: Profiles for temperature and power during microwave irradiation in DYN (top) and SPS (bottom) modes.
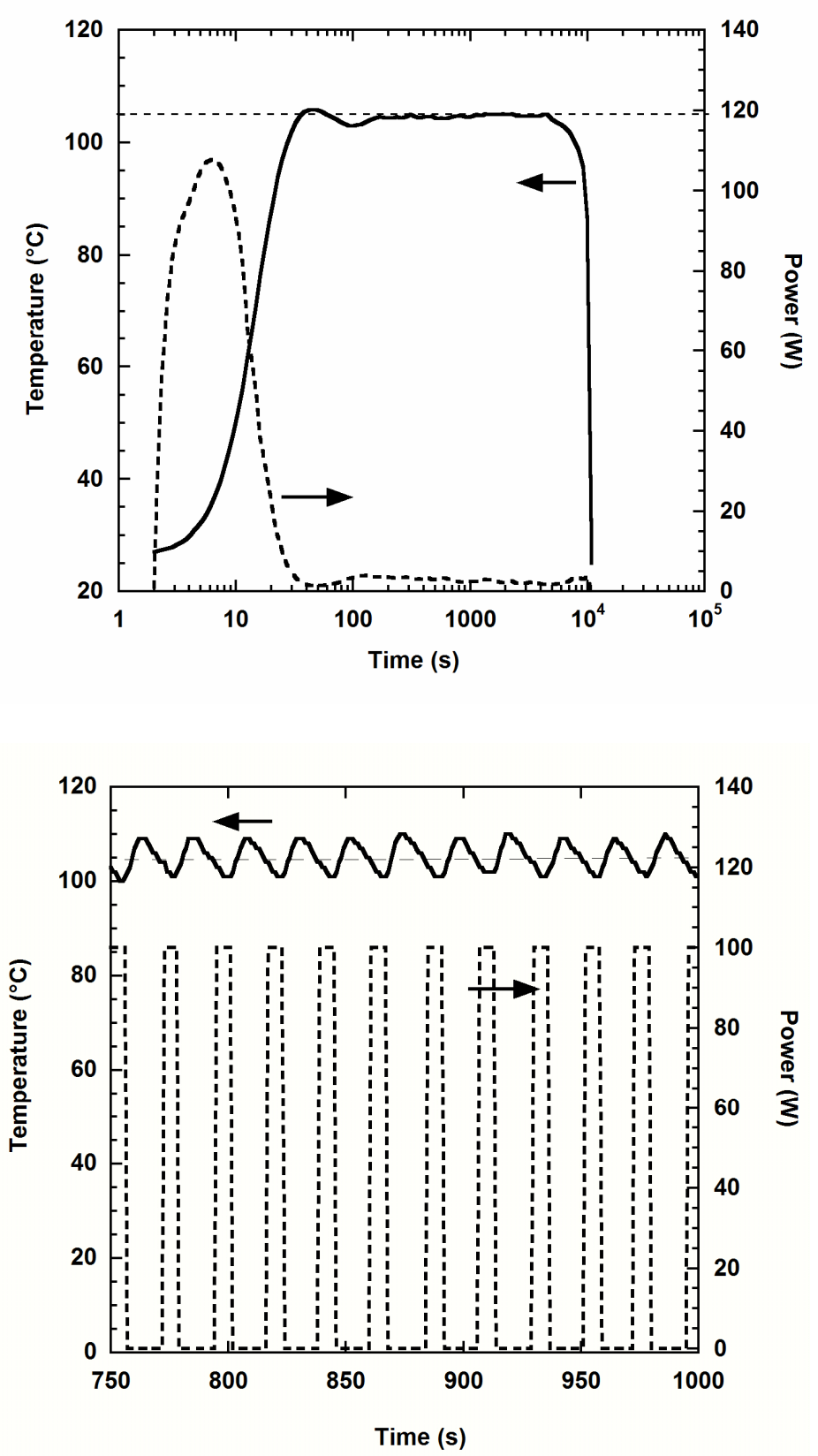


\section{Results and discussions}

\subsection{Conventional heating $(\mathrm{CH})$ versus dynamic mode (DYN)}

Recently, Nicolay et $a l^{32}$ and Phan et $a l^{33}$ demonstrated that the polymerization in homogeneous aqueous solution was possible below the boiling point of water by using second-generation nitroxides, namely $\quad N$-tert-butyl- $N$-(1-diethylphosphono-2,2dimethylpropyl) nitroxide (SG1). SG1 exhibits a higher activation/deactivation equilibrium constant than TEMPO as well as a good solubility in concentrated solutions of water-soluble monomers (> $10^{-2} \mathrm{~mol} / \mathrm{L}$ in $40 \mathrm{wt}-\%$ of acrylamide, for example). Otherwise, very few watersoluble alkoxyamines have been described, and a majority of studies concerning NMPs in dispersed aqueous media have involved bicomponent or monocomponent initiating systems. $^{34-37}$ Recently, it was shown that reasonably controlled nitroxide-mediated polymerization of acrylamide could be achieved in a pure water solution at $120^{\circ} \mathrm{C}$ and high monomer concentration (40 wt\%), using a bicomponent inititating system, i.e., a conventional hydrosoluble radical initiator (Vazo56) in combination with SG1. ${ }^{27}$ The strategy adopted to prevent the loss of control of the polymerization and its lack of reproducibility involves adding an excess of scavenging nitroxide before triggering the polymerization. Usually in NMP, the amount of free nitroxide radicals present at the NMP equilibrium can be calculated from the equilibrium constant between active and dormant chains, $\mathrm{K}=\mathrm{k}_{\mathrm{d}} / \mathrm{k}_{\mathrm{c}}$, the rate constant of self-termination, $\mathrm{kt}$, and the rate constant of propagation, $\mathrm{kp}:{ }^{38}$

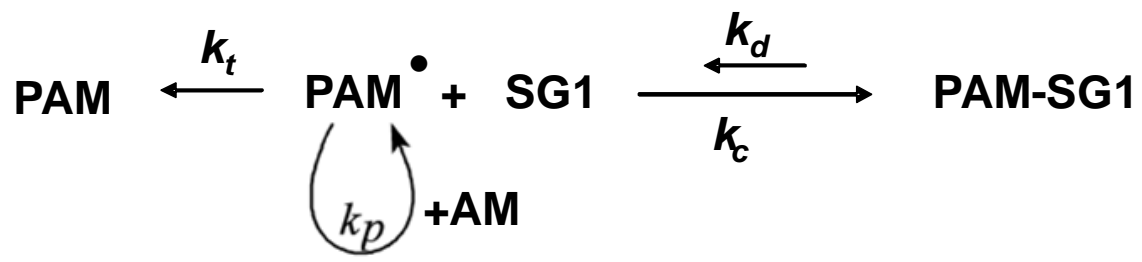

Since these constants were unknown, the experimental conditions were determined according to previous work: ${ }^{27}$ the molar ratio $\mathrm{r}=[\mathrm{AM}]_{0} / 2[\mathrm{Vazo} 56]=500$ for a constant acrylamide $\mathrm{AM}$ concentration of $40 \mathrm{wt} \%$ in water and a constant molar ratio [SG1 $]_{0} /[\text { Vazo56 }]_{0}$ of 1.8 . These conditions were far from optimized, but nevertheless, with such a system, a good compromise between kinetics and polymerization control was obtained, as can be seen from the discussion below.

Figure 3 displays the kinetic results and the living character of the polymerization for the $\mathrm{CH}$ and DYN modes, within a temperature range of 90 to $120^{\circ} \mathrm{C}$ (Table 1). The concentration of 
active species remained constant regardless of the experimental conditions as evidenced from the straight semi-logarithmic plots (Figure 3a). Moreover, neither an induction period nor a steepening with the increasing conversion could be observed. Control over the growth of polymer chains was furthermore shown by the straight line of the molar mass versus polymerization yield (Figure 3b) along with unimodal aqueous SEC chromatograms. The polydispersities followed the expected trend ${ }^{39}$ and decreased with increasing conversion, with a final $\mathrm{Mw} / \mathrm{Mn}<1.1$ for all studied polymerizations. The downward curvature that was observed at low conversion in Figure $3 \mathrm{~b}$ could not be attributed to the SEC separation since the MALS detector provides absolute molar masses. A lack of linearity at the early stage of the polymerization may be discussed. One possible reason could be a slow initiation resulting in the formation of fewer chains at low conversion. In the case of the azo-initiator, the production rate of primary radicals was dominated by the thermal decomposition and the initiator efficiency can be defined as the fraction of radicals produced in the homolysis reaction initiating polymer chains. The disappearance rate of the initiator can be expressed in terms of the initiator half time $\left(\mathrm{t}_{1 / 2}\right)$, defining the time it takes for the concentration of the initiator to reach half of its original value. The initiator half time is temperature dependant and, for VAZO-56, it was calculated to a value of 9 minutes at $90^{\circ} \mathrm{C}$ and 109 seconds at $105^{\circ} \mathrm{C} .{ }^{40}$ However, this was not obvious from the kinetic plots as no temperature effect could be observed in Figure $3 \mathrm{~b}$ and no upward curvature indicating an increase in [PAM ${ }^{\bullet}$ ] occurring in case of slow initiation is observed in Figure 3a. 


\section{Figure 3:}

a) Kinetic plots of $\ln \left([\mathrm{AM}]_{0} /[\mathrm{AM}]\right)$ vs. reaction time for the NMP polymerization of acrylamide under conventional heating $(\mathrm{CH})$ and dynamic microwave irradiation (DYN) at various temperatures: $\mathrm{CH}$ (full symbols), $120{ }^{\circ} \mathrm{C}(\boldsymbol{\bullet}), 115^{\circ} \mathrm{C}(\boldsymbol{\square}), 105^{\circ} \mathrm{C}(\bullet), 90^{\circ} \mathrm{C}(\boldsymbol{\nabla})$; DYN (open symbols), $105^{\circ} \mathrm{C}(\diamond), 97^{\circ} \mathrm{C}(\triangle)$ and $90^{\circ} \mathrm{C}(\nabla)$.

b)The dependence of the number-average molar mass $\left(M_{n}\right)$ and the polydispersity index (Ip = $\mathrm{Mw} / \mathrm{Mn}$ ), on the monomer conversion, p. Same symbols as in (a). The dashed lines are guides for the eyes.
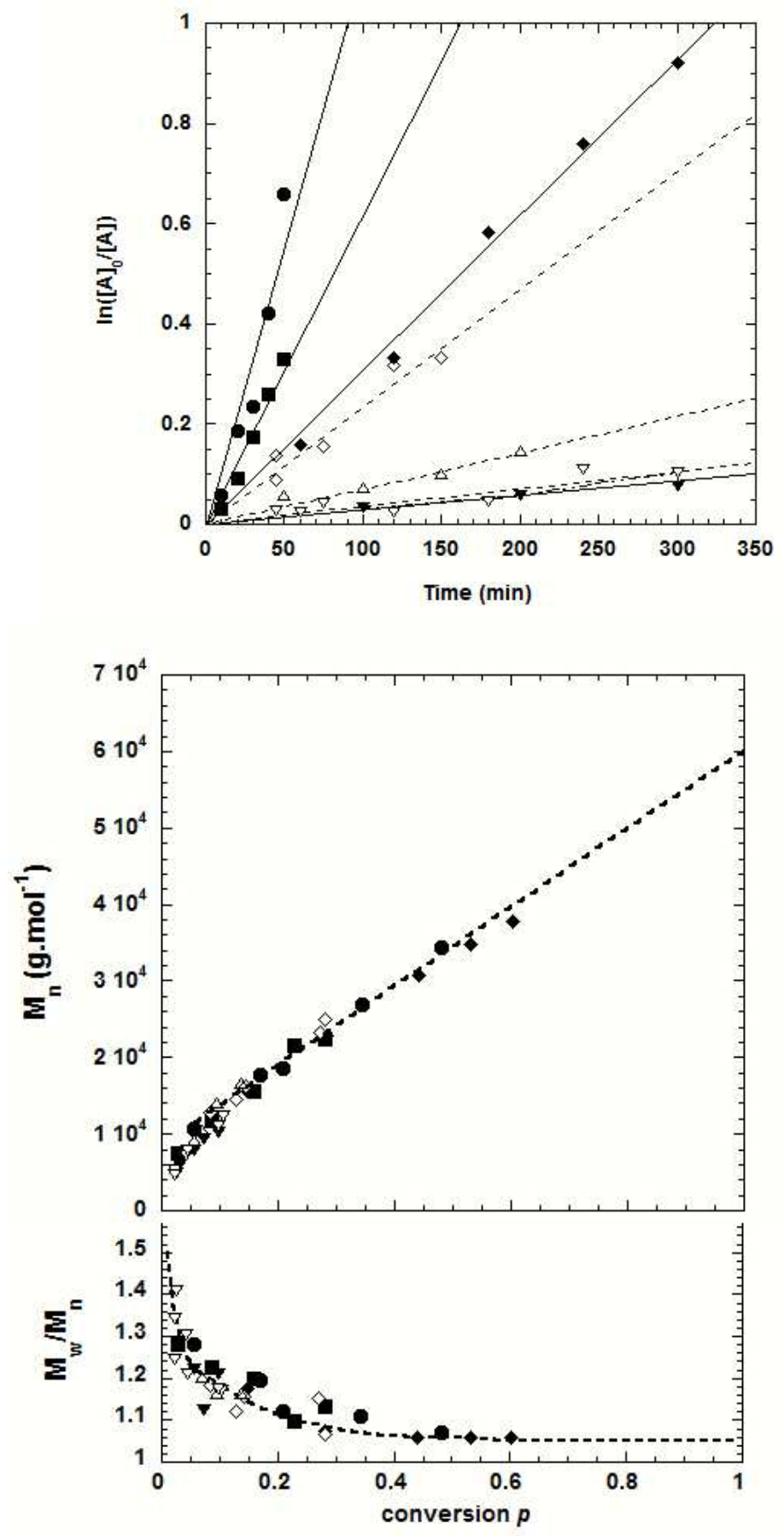
Table 1 : Temperature $(\mathrm{T})$, polymer conversion $(\mathrm{p})$, reaction time $(\mathrm{t})$, number-average molar mass $\left(\mathrm{M}_{\mathrm{n}}\right)$ and polydispersity index (Ip) of the NMP of acrylamide in aqueous solution using conventional heating $(\mathrm{CH})$ and two microwave modes (DYN and SPS): $r=[A M] / 2[$ Vazo56] $=500$ for a constant acrylamide (AM) concentration $(40 \mathrm{wt} \%)$ and a constant molar ratio $[\mathrm{SG} 1]_{0} /[\mathrm{Vazo} 56]_{0}=1.8$.

\begin{tabular}{|c|c|c|c|c|c|c|c|c|c|c|c|c|c|c|c|c|}
\hline \multicolumn{5}{|c|}{$\mathrm{CH}$} & \multicolumn{5}{|c|}{ DYN } & \multicolumn{7}{|c|}{ SPS } \\
\hline $\begin{array}{c}\mathrm{T} \\
\left({ }^{\circ} \mathrm{C}\right) \\
\end{array}$ & $\begin{array}{c}\mathrm{t} \\
(\mathrm{min})\end{array}$ & $\mathrm{p}$ & $\begin{array}{c}\mathrm{Mn} \\
\left(\mathrm{g} \cdot \mathrm{mol}^{-1}\right)\end{array}$ & Ip & $\begin{array}{c}\mathrm{T} \\
\left({ }^{\circ} \mathrm{C}\right)\end{array}$ & $\begin{array}{c}\mathrm{t} \\
(\mathrm{min})\end{array}$ & $\mathrm{p}$ & $\begin{array}{c}\mathrm{Mn} \\
\left(\mathrm{g} \cdot \mathrm{mol}^{-1}\right)\end{array}$ & Ip & $\begin{array}{c}\mathrm{T} \\
\left({ }^{\circ} \mathrm{C}\right)\end{array}$ & $\begin{array}{c}\mathrm{t} \\
(\mathrm{min})\end{array}$ & $\begin{array}{c}\mathrm{P} \\
(\mathrm{W})\end{array}$ & $\begin{array}{l}\mathrm{E}_{\mathrm{MW}} \\
(\mathrm{kJ})\end{array}$ & $\mathrm{p}$ & $\begin{array}{c}\mathrm{Mn} \\
\left(\mathrm{g} \cdot \mathrm{mol}^{-1}\right)\end{array}$ & Ip \\
\hline \multirow[t]{6}{*}{120} & 10 & 0.056 & 10700 & 1.28 & 105 & 45 & 0.084 & 12900 & 1.18 & 105 & 60 & 10 & 35 & 0.16 & 16400 & 1.23 \\
\hline & 20 & 0.170 & 17700 & 1.20 & & 45 & 0.128 & 14600 & 1.12 & & 60 & 50 & 89 & 0.90 & 59000 & 1.18 \\
\hline & 30 & 0.210 & 18600 & 1.12 & & 75 & 0.143 & 16300 & 1.16 & & 60 & 100 & 116 & 0.97 & 59800 & 1.15 \\
\hline & 40 & 0.344 & 27000 & 1.11 & & 120 & 0.271 & 23200 & 1.15 & & 60 & 150 & 133 & 0.99 & 60000 & 1.20 \\
\hline & 50 & 0.482 & 34400 & 1.07 & & 150 & 0.282 & 25000 & 1.07 & & 60 & 200 & 142 & 0.99 & 60100 & 1.24 \\
\hline & & & & & & & & & & & 60 & 250 & 156 & 0.99 & 61700 & 1.20 \\
\hline \multirow[t]{6}{*}{115} & 10 & 0.029 & 7450 & 1.28 & 97 & 50 & 0.056 & 9150 & 1.29 & & 60 & 300 & 168 & 0.99 & 61500 & 1.15 \\
\hline & 20 & 0.087 & 11800 & 1.23 & & 100 & 0.069 & 10400 & 1.20 & & & & & & & \\
\hline & 30 & 0.159 & 15600 & 1.20 & & 150 & 0.096 & 14100 & 1.16 & 90 & 30 & 150 & 42 & 0.10 & 15800 & 1.40 \\
\hline & 40 & 0.227 & 21600 & 1.10 & & 200 & 0.136 & 16700 & 1.16 & & 30 & 190 & 49 & 0.26 & 35400 & 1.27 \\
\hline & 50 & 0.281 & 22500 & 1.13 & & & & & & & 60 & 50 & 60 & 0.05 & 8430 & 1.26 \\
\hline & & & & & 90 & 45 & 0.027 & 5020 & 1.41 & & 60 & 100 & 72 & 0.11 & 13900 & 1.20 \\
\hline \multirow[t]{6}{*}{105} & 60 & 0.147 & 15500 & 1.18 & & 60 & 0.023 & 5380 & 1.24 & & 60 & 150 & 81 & 0.18 & 19600 & 1.18 \\
\hline & 120 & 0.282 & 22800 & 1.07 & & 75 & 0.041 & 7160 & 1.31 & & 60 & 160 & 84 & 0.33 & 24000 & 1.23 \\
\hline & 180 & 0.441 & 30800 & 1.06 & & 120 & 0.024 & 4660 & 1.34 & & 60 & 170 & 86 & 0.34 & 23300 & 1.22 \\
\hline & 240 & 0.532 & 34800 & 1.06 & & 180 & 0.046 & 8000 & 1.21 & & 60 & 180 & 87 & 0.23 & 22000 & 1.28 \\
\hline & 300 & 0.602 & 37700 & 1.06 & & 240 & 0.104 & 12400 & 1.17 & & 60 & 190 & 118 & 0.94 & 60000 & 1.16 \\
\hline & & & & & & 300 & 0.098 & 11100 & 1.18 & & 60 & 200 & 119 & 0.94 & 56700 & 1.29 \\
\hline \multirow[t]{8}{*}{90} & 100 & 0.032 & 5940 & 1.29 & & & & & & & 60 & 250 & 123 & 0.95 & 62700 & 1.18 \\
\hline & 200 & 0.055 & 8000 & 1.22 & & & & & & & 120 & 150 & 196 & 0.95 & 59600 & 1.19 \\
\hline & 300 & 0.098 & 10300 & 1.21 & & & & & & & 180 & 150 & 280 & 0.98 & 60600 & 1.19 \\
\hline & 300 & 0.073 & 9350 & 1.12 & & & & & & & 240 & 150 & 377 & 0.99 & 63600 & 1.14 \\
\hline & & & & & & & & & & & $300^{\mathrm{a}}$ & 150 & 450 & 0.99 & 60400 & 1.15 \\
\hline & & & & & & & & & & 80 & 60 & 200 & 64 & 0.05 & 8330 & 1.34 \\
\hline & & & & & & & & & & & 60 & 250 & 74 & 0.10 & 14600 & 1.37 \\
\hline & & & & & & & & & & & 60 & 300 & 76 & 0.19 & 15500 & 1.37 \\
\hline
\end{tabular}

a) used for chain extension polymerization

As expected, the kinetics depended on the temperature (Figure 3a) and the polymerization rate was significantly higher at $120{ }^{\circ} \mathrm{C}$ than at $90{ }^{\circ} \mathrm{C}$. Indeed, the cleavage rate constant, $k_{\mathrm{d}}$, depended on the dissociation energy of the alkoxyamine bond as well as the Arrhenius temperature, whereas the cross-combination constant, $k_{\mathfrak{c}}$, demonstrated non-Arrhenius temperature dependencies. ${ }^{41}$ The time dependence could be approximated as first-order over a 
significant range of the reaction time: $\ln ([A M] d[A M])=k_{\text {app }}$.t. The obtained values for $\mathrm{k}_{\mathrm{app}}$ (in $\mathrm{s}^{-1}$ ) are reported in Figure 4 where they are also plotted versus $1 / \mathrm{T}$ according to the following Arrhenius relation,

$$
\mathrm{k}_{\mathrm{app}}=\mathrm{A} \exp \left(-\mathrm{E}_{\mathrm{A}} / \mathrm{RT}\right) \text {. }
$$

Here, $\mathrm{E}_{\mathrm{A}}$ is the apparent activation energy of the polymerization and $\mathrm{R}$ is the universal gas constant.

Figure 4 clearly shows that both the $\mathrm{CH}$ and DYN modes presented similar $E_{A}$ values. Moreover, the value $\mathrm{E}_{\mathrm{A}}=140 \mathrm{KJ} / \mathrm{mol}$, was of the same order of magnitude as that usually obtained in NMP. ${ }^{38}$ This thus demonstrated the absence of specific MW effects in the case of the DYN mode.

While the plots at $90^{\circ} \mathrm{C}$ obtained by both the DYN and $\mathrm{CH}$ modes were in good agreement, a deviation was noticed at $105^{\circ} \mathrm{C}$ and was attributed to the experimental errors that were also present in Figure 3a. The absence of MW effects can be explained by the DYN parameters themselves, i.e., a high microwave power at the beginning of the polymerization increasing the bulk temperature to the set point as quickly as possible. Upon reaching the target temperature, the microwave power was decreased or completely shut off in order to maintain the desired bulk temperature, $T_{p}$, without exceeding it (Figure 2a). In the absence of microwave irradiation, classical thermal chemistry takes over, and the full advantage of microwave accelerated synthesis is lost.

Figure 4: An Arrhenius plot of the NMP of acrylamide under conventional heating $(\mathrm{CH}$, and dynamic microwave irradiation (DYN, O).

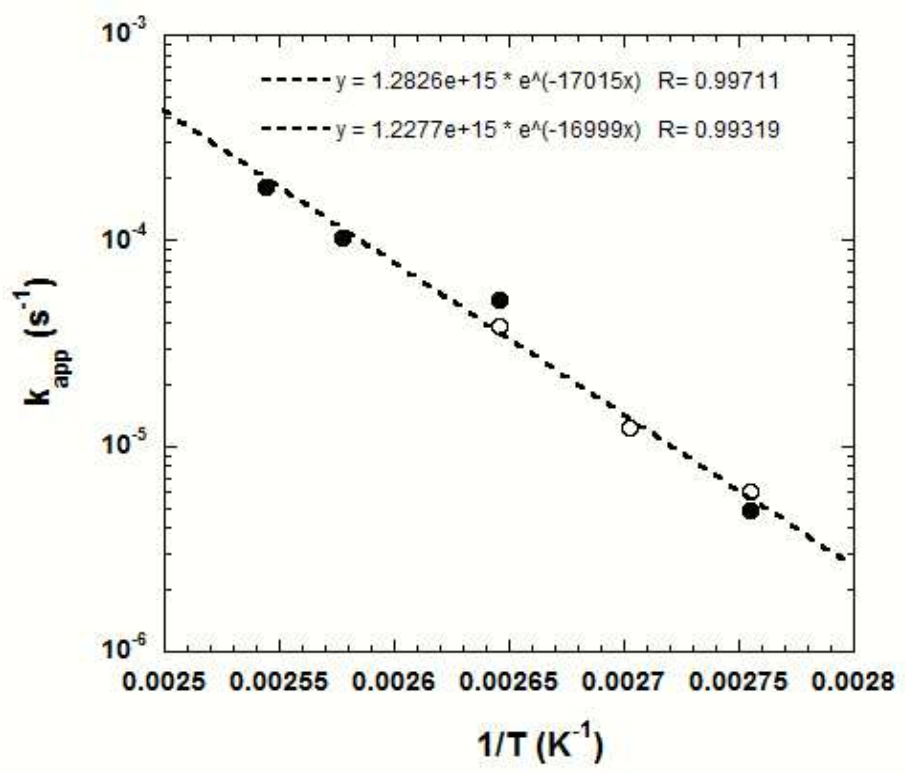




\subsection{Pulsed mode (SPS)}

To ensure that a high and constant level of microwave energy is applied during the polymerization, the previously described pulsed mode (SPS) was utilized. As opposed to the DYN mode, the reaction vessel is, in SPS mode, externally cooled with compressed air, while microwave irradiation is simultaneously administered. This way, more energy can be directly applied to the reaction mixture. With DYN, microwave irradiation is predominantly used to reach $T_{P}$ faster. SPS mode polymerizations were performed during one hour at 80, 90 and 105 ${ }^{\circ} \mathrm{C}$ and the MW power ranged from 10 to $300 \mathrm{~W}$ (Table 1). Consequently, the MW energy varied from 10 to $170 \mathrm{~kJ}$ for a constant volume of reaction mixture $(3 \mathrm{~mL})$. Figure 5 shows the polymer conversion, $\mathrm{p}$, obtained after one hour according to $\mathrm{E}_{\mathrm{MW}}$. At low $\mathrm{E}_{\mathrm{MW}}$, the conversion increased gradually up to a point of MW energy where the conversion displayed a jump. After this specific point, the conversion once again increased gradually up to complete conversion of the monomer. It seemed that this specific microwave effect was independent of the temperature and occurred at an $\mathrm{E}_{\mathrm{MW}}$ of around $90 \mathrm{~kJ}$.

Figure 5: The polymer conversion as a function of the microwave energy during one hour of the NMP of acrylamide under SPS microwave irradiation at various temperatures: $105^{\circ} \mathrm{C}(\boldsymbol{\Delta})$, $90^{\circ} \mathrm{C}(\boldsymbol{\square})$ and $80^{\circ} \mathrm{C}$

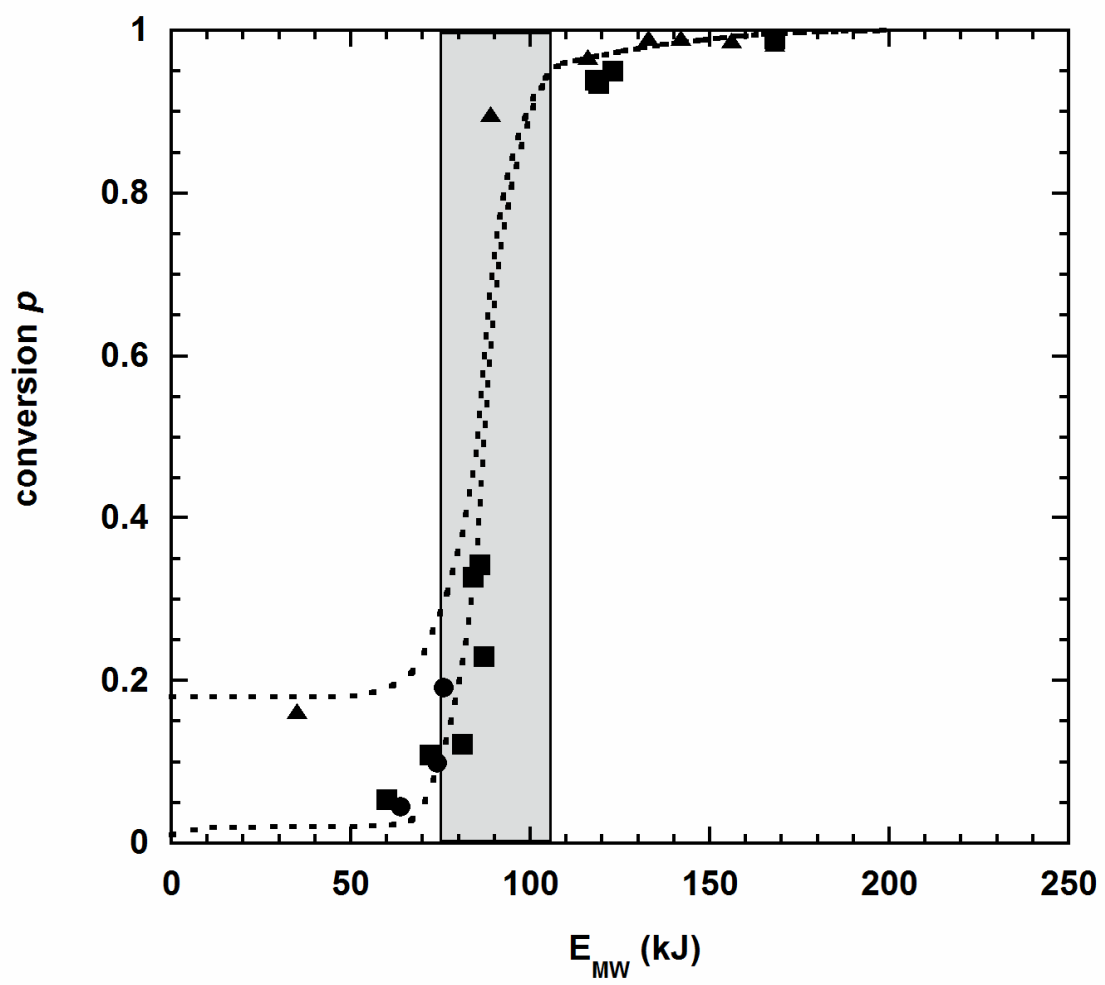


This series of experiments (carried out at a constant reaction time) was completed by two series performed at constant power $(150$ and $190 \mathrm{~W})$ and temperature $\left(90^{\circ} \mathrm{C}\right)$. In these cases, the reaction time was the only variable parameter, i.e., the microwave energy. At 190W and $150 \mathrm{~W}$, the polymer conversion increased sharply between 50 and $120 \mathrm{~kJ}$ (Table 1). This could correspond to a lowering of the concentration of nitroxide radicals in the medium. As shown in Figure 6, the variation of $\ln \left([\mathrm{AM})_{0} /[\mathrm{AM}]\right)$ vs time was not linear, thereby suggesting a loss of the "living character" of the polymerization. However, the control over the growth of polymer chains was evidenced by the linear evolution of molar masses with conversion as well as an acceptable polydispersitiy index below 1.3 and unimodal aqueous SEC chromatograms. Figure 7 shows this linear evolution of molar masses, and as can be evidenced, there was a perfect match to the results previously obtained in DYN and $\mathrm{CH}$ modes. One of the differences between the DYN and SPS modes was the use of air cooling during the latter. This is known to give rise to a significant underestimation of the temperature readout of the IR sensor since the air cools mainly the exterior of the reaction vessel. The effect that the temperature may have on the auto-acceleration polymerization is discussed in the following section.

Figure 6: Kinetic plots of $\ln \left([\mathrm{AM}]_{0} /[\mathrm{AM}]\right)$ (full symbols) and polymer conversion, $p$ (open symbols) vs. the reaction time for NMP of acrylamide under pulsed microwave irradiation (SPS) at $90^{\circ} \mathrm{C}$ and varying power: $150 \mathrm{~W}$ (ring) and $190 \mathrm{~W}$ (square).

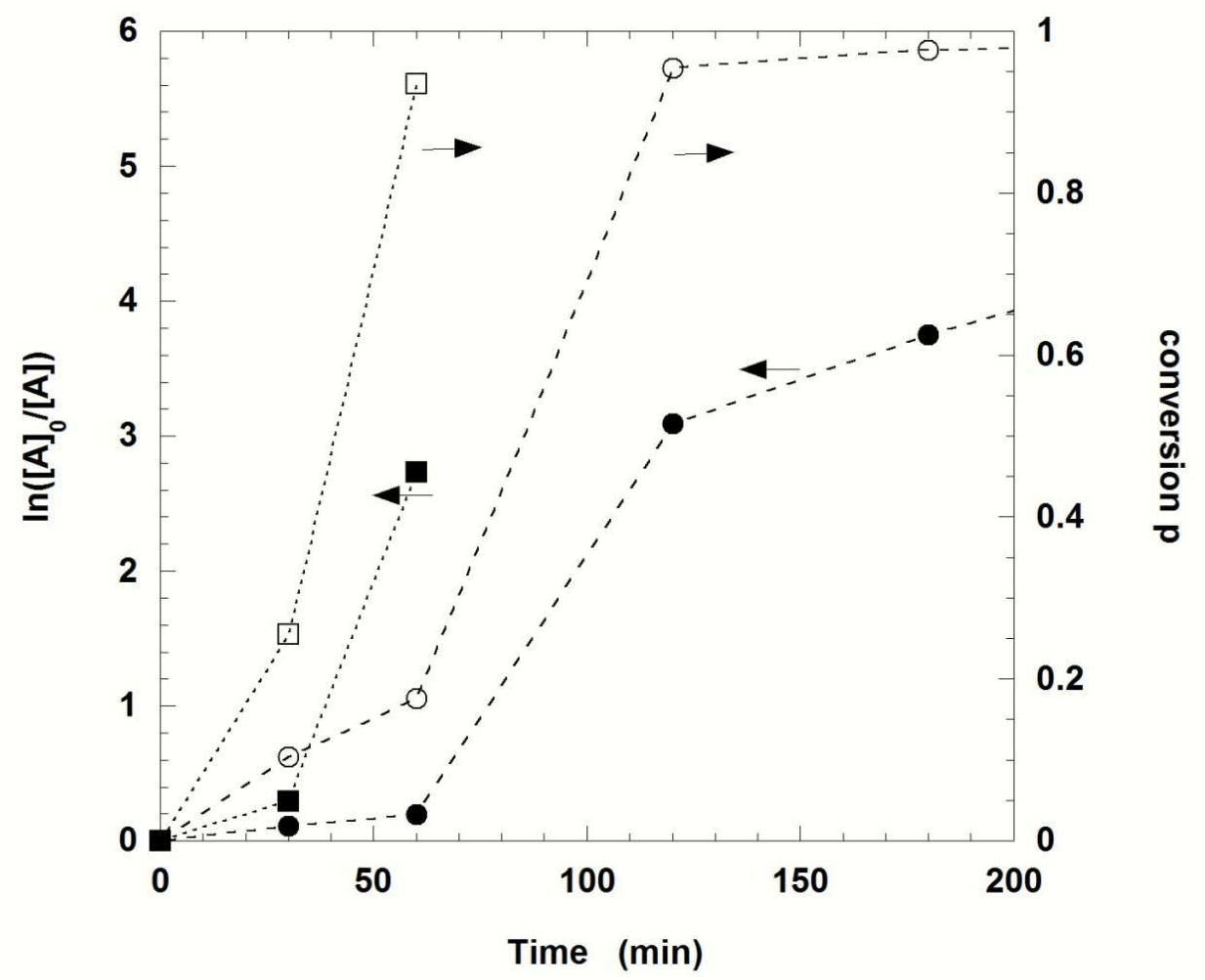


Figure 7: The number-average molar mass $\left(M_{n}\right)$, and the polydispersity index $(\mathrm{Ip}=\mathrm{Mw} / \mathrm{Mn})$, on the monomer conversion, $\mathrm{p}$, for NMP of acrylamide under pulsed microwave irradiation (SPS) at $105^{\circ} \mathrm{C}(\boldsymbol{\Delta}), 90^{\circ} \mathrm{C}(\boldsymbol{\square})$ and $80^{\circ} \mathrm{C}(\boldsymbol{O})$ for the runs presented in figure 5 and 6 (the dashed lines provide the variation observed in Figure 3 for $\mathrm{CH}$ and DYN mode).

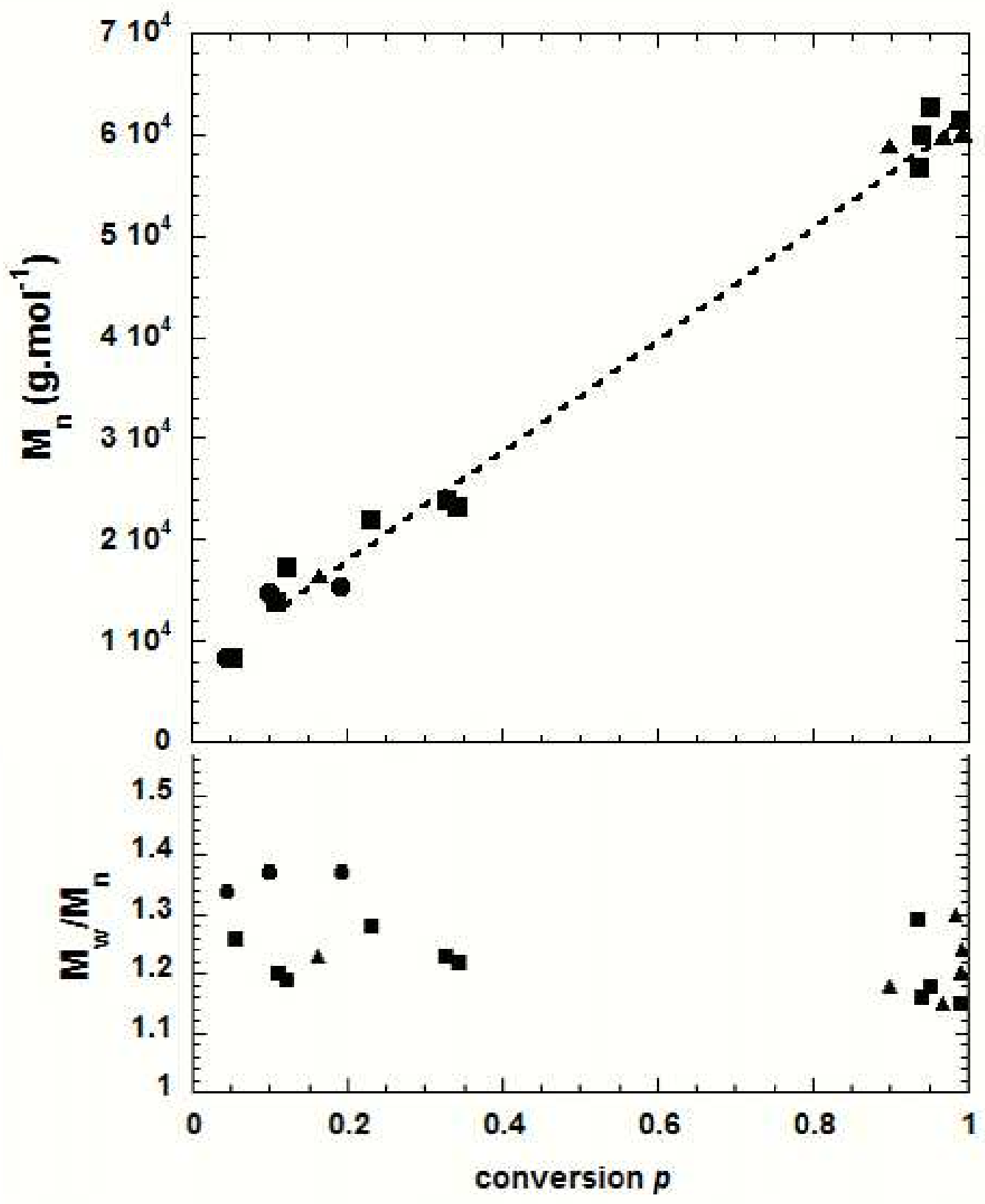

The living character of the PAM polymer chains produced with the vazo-SG1 system has been further confirmed by chain extension experiments with a second addition of the same AM monomer. The unpurified PAM formed at high conversion ( $\mathrm{p}=99 \%)$ from aqueous NMP in SPS mode $\left(90^{\circ} \mathrm{C}, 150 \mathrm{~W}, 300 \mathrm{~min}\right.$, cf. Table 1) was used as a macroinitiator for further chain extension $(\mathrm{Mn}=60400 \mathrm{~g} / \mathrm{mol}$ and $\mathrm{Ip}=1.15)$. Two chain extension polymerizations, denoted E1 and E2, were carried out by a second addition of acrylamide to maintain the total 
concentration of monomer plus macroinitiator at $40 \mathrm{wt} \%$. For E1 and E2, respectively $0.5 \mathrm{~g}$ and $1.16 \mathrm{~g}$ of macroinitiator solution were completed up to $3 \mathrm{~g}$ with a $40 \mathrm{wt} \%$ of acrylamide solution. All other parameters and procedures were the same as in the NMP procedure in SPS mode. E1 and E2 were irradiated $120 \mathrm{~min}$ at $90^{\circ} \mathrm{C}$ under $100 \mathrm{~W}$ and $60 \mathrm{~min}$ at $90^{\circ} \mathrm{C}$ under 190W, respectively. The conversions measured by CES were found to be $6 \%$ and $42 \%$ for respectively E1 and E2, which corresponded to the targeted PAM molar masses of 77700 and $100400 \mathrm{~g} / \mathrm{mol}$. The $\mathrm{M}_{\mathrm{n}}$ and Ip of the chain-extended polymer were increased up to 76400 and $97600 \mathrm{~g} \mathrm{~mol}^{-1}$, with Ip $=1.17$ and 1.25, respectively for E1 and E2. These results indicate that the PAM homopolymer, used as the macroinitiator, was effectively functionalized with nitroxide end groups. After the chain extension experiment, the molar mass distribution of the chain-extended product shifted to higher molar masses as compared to that of the initial PAM macroalkoxyamine (Figure 8). These experimental results exhibited a good control of PAM molar masses even at very high polymer conversion, i.e. even close to $100 \%$, by Nitroxide Mediated polymerization under microwave irradiation in aqueous medium.

Figure 8: Molar mass distributions, differential (dashed line) and cumulative (continuous line), for chain extension polymerization: macroinitiator, E1 and E2 (from left to right).

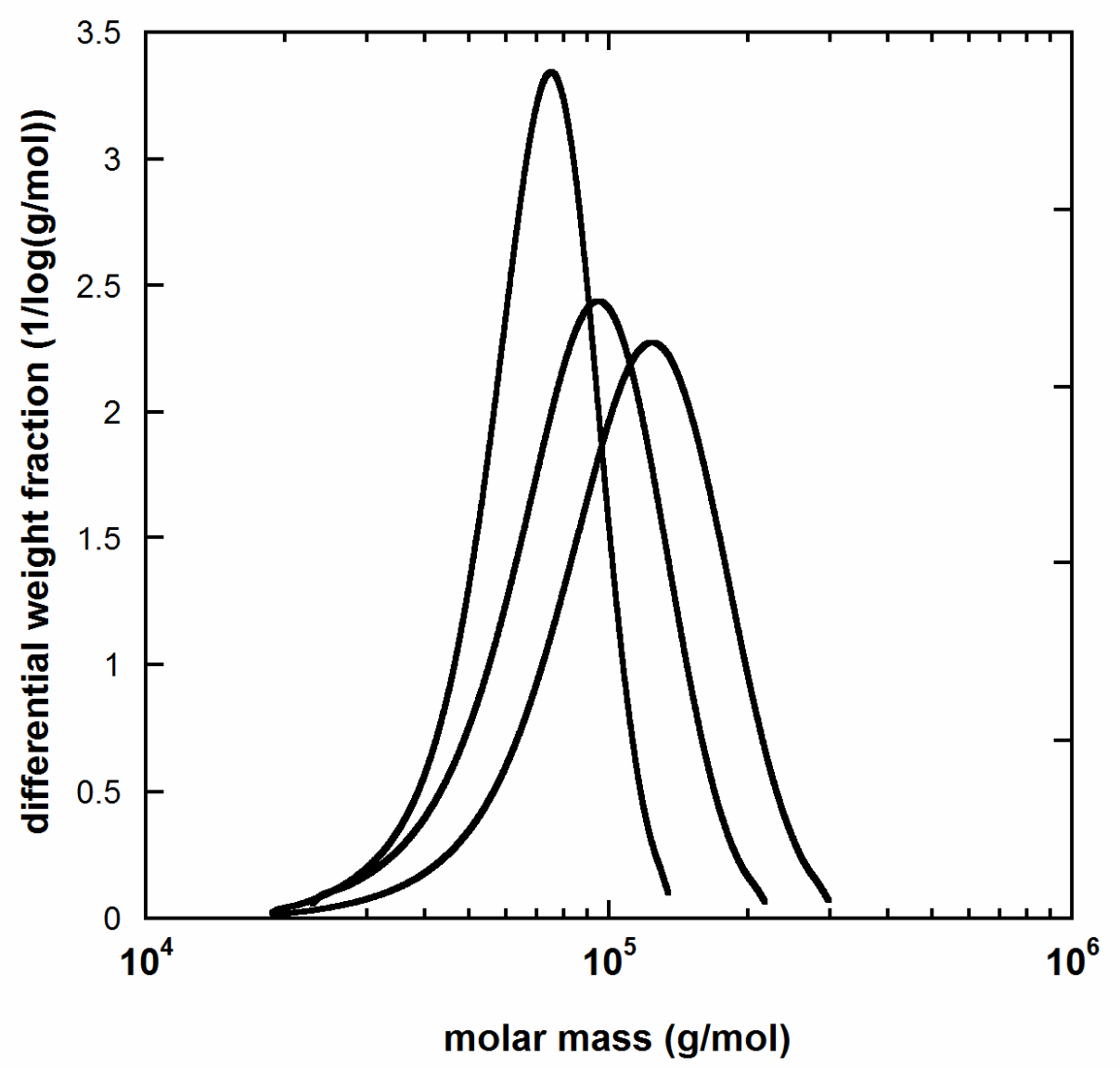




\subsection{Specific microwave effect}

Despite the relatively large number of consistent publications in the area of microwaveassisted organic/polymer chemistry, the exact reasons why microwave irradiation is able to enhance chemical processes remain unknown. ${ }^{2,4}$ Since the start of microwave synthesis, rateaccelerations and occasional variations in product distributions as compared to experiments by conventional heating have led to speculations regarding the existence of so-called "nonthermal" or "specific" microwave effects. According to investigations by Kappe, Loupy ${ }^{4}$ and coworkers, it can be understood that the differences between "nonthermal" and "specific" microwave effects depend on the scale of the observation, which is either micro- or macroscopic. The existence of so-called "specific microwave effects" is in principle undisputed since they cannot be reproduced by conventional heating. They should thus be the result of the uniqueness of the dielectric phenomenon in microwave heating, i) the superheating effect of solvents at the atmospheric pressure; ii) the selective heating such as the strong microwave absorption of the heterogeneous catalysts or reagents in a less polar medium of reaction; and iii) the elimination of the wall effects caused by the inverted temperature gradients. ${ }^{2}$ In contrast, "nonthermal microwave effects" represent a controversial subject and have been suggested to result from a direct interaction - which is unrelated to a macroscopic temperature effect - of the electric field with specific molecules in a reaction medium ${ }^{4}$. In our work, the intention was not to enhance the microwave-heated reaction with a high temperature reaction that can rapidly be attained when irradiating polar materials/reaction mixtures under closed-vessel conditions in a microwave field. The microwave-assisted polymerization in an aqueous solvent was carried out at a temperature lower than $105^{\circ} \mathrm{C}$ and at low pressure. It is worth noticing that the experimental conditions used within this study were far from the near-critical or supercritical water condition ${ }^{23}$ which involves $\mathrm{T}>150^{\circ} \mathrm{C}$, pressure $>4$ bar; $\mathrm{T}>374^{\circ} \mathrm{C}, \mathrm{p}>200$ bar.

A microwave enhancement of chemical reactions takes place only during the application of microwave energy. This energy source provides a direct activation of the molecules in a chemical reaction, for which reason it would be undesirable to suppress its application as in the DYN mode. SPS ensures the application of a high and constant level of microwave energy. It was previously demonstrated that the experiments conducted with the DYN and $\mathrm{CH}$ modes were in good agreement and revealed no specific MW effects. The polymerization performed in SPS mode, on the other hand, exhibited a sharp increase in polymer conversion within 1 hour (Figure 5). As a comparison, the conversion, p, was lower than 0.5 for one hour 
of reaction at $120^{\circ} \mathrm{C}$ in $\mathrm{CH}$ mode and below 0.3 at $105^{\circ} \mathrm{C}$ in the $\mathrm{DYN}$ and $\mathrm{CH}$ modes, i.e., at least $15^{\circ} \mathrm{C}$ above the temperature used in the SPS mode (extrapolated data of figure 3). The temperature control as measured by IR sensors $\left( \pm 4^{\circ} \mathrm{C}\right)$ was the same in both MW modes and could not explain the auto-acceleration polymerization. In fact, the use of IR sensors may be inappropriate for temperature measurements in microwave-heated reactions. The reason for this is that they can easily lead to a misinterpretation of results since the real reaction temperatures are unknown, ${ }^{2}$ in particular for SPS modes where external cooling can underestimate the reaction temperature. To specify the origin of the auto-acceleration polymerization, a complementary polymerization series at $80^{\circ} \mathrm{C}$ was carried out only in SPS mode $(\mathrm{P}=250 \mathrm{~W})$, during which the viscosity of each sample was measured and the pressure in the vessel was monitored.

In order for the results to be as reliable as possible, the same reaction vessels, reaction volumes and stirring (speed and magnets) were used in all experiments. Consequently, all parameters were identical apart from the time of irradiation, and therefore, a fair comparison based on the polymerization times and conversion could be carried out for the experiments performed solely in the SPS mode. Figure 9 presents a superposition of the kinetic plot results and the viscosity, and the inset displays the linear evolution of the molar masses according to the results previously discussed. As can be seen, the sharp rate enhancement of the acrylamide NMP appeared at $240 \min \left(\mathrm{p}=0.5\right.$ and $\left.\mathrm{E}_{\mathrm{MW}}>90 \mathrm{~kJ}\right)$ and at the same time, the viscosity displayed a significant increase without raising the pressure inside the vessel. It was possible that the underestimation of the temperature reaction due to the IR sensors was even stronger after gelation of the polymerization mixture since the heat transport to the vessel wall worsened. This could explain the observation of a sudden increase in conversion. However, the auto-accelaration polymerization was not followed by a significant increase in pressure within the vessel, according to the precision of the pressure sensor as shown in Figure 9. It could thereby be assumed that the reaction temperature did not increase excessively after the gel point. As shown in Figures 6 and 9, the apparent constant rate of the auto-acceleration polymerization was estimated to be higher than $710^{-4} \mathrm{~s}^{-1}$, corresponding to a theoretical temperature above $130^{\circ} \mathrm{C}$ according to the Arrhenius relation established previously.

An underestimation of the reaction temperature medium at the gel point could thus not be neglected. However this cannot be the sole explanation behind the auto-acceleration polymerization. Unfortunately, the CEM microwave reactor in the present study did not allow 
the measurement of the real temperature inside the vessel during the SPS mode as a quantification of the reliability of the IR sensor.

Figure 9: Kinetic plots of $\ln \left([\mathrm{AM}]_{0} /[\mathrm{AM}]\right)$ vs. reaction time for NMP of acrylamide $(\boldsymbol{\square})$ and the corresponding viscosity of the reaction mixture $(\mathbf{A})$, under pulsed microwave irradiation at $80^{\circ} \mathrm{C}$ and $250 \mathrm{~W}$. Inset: the dependence of the number-average molar mass, $\mathrm{Mn}$, on the monomer conversion, p ( ). The lines are guides for the eyes. Below: superposition of measured pressures for each experiment.

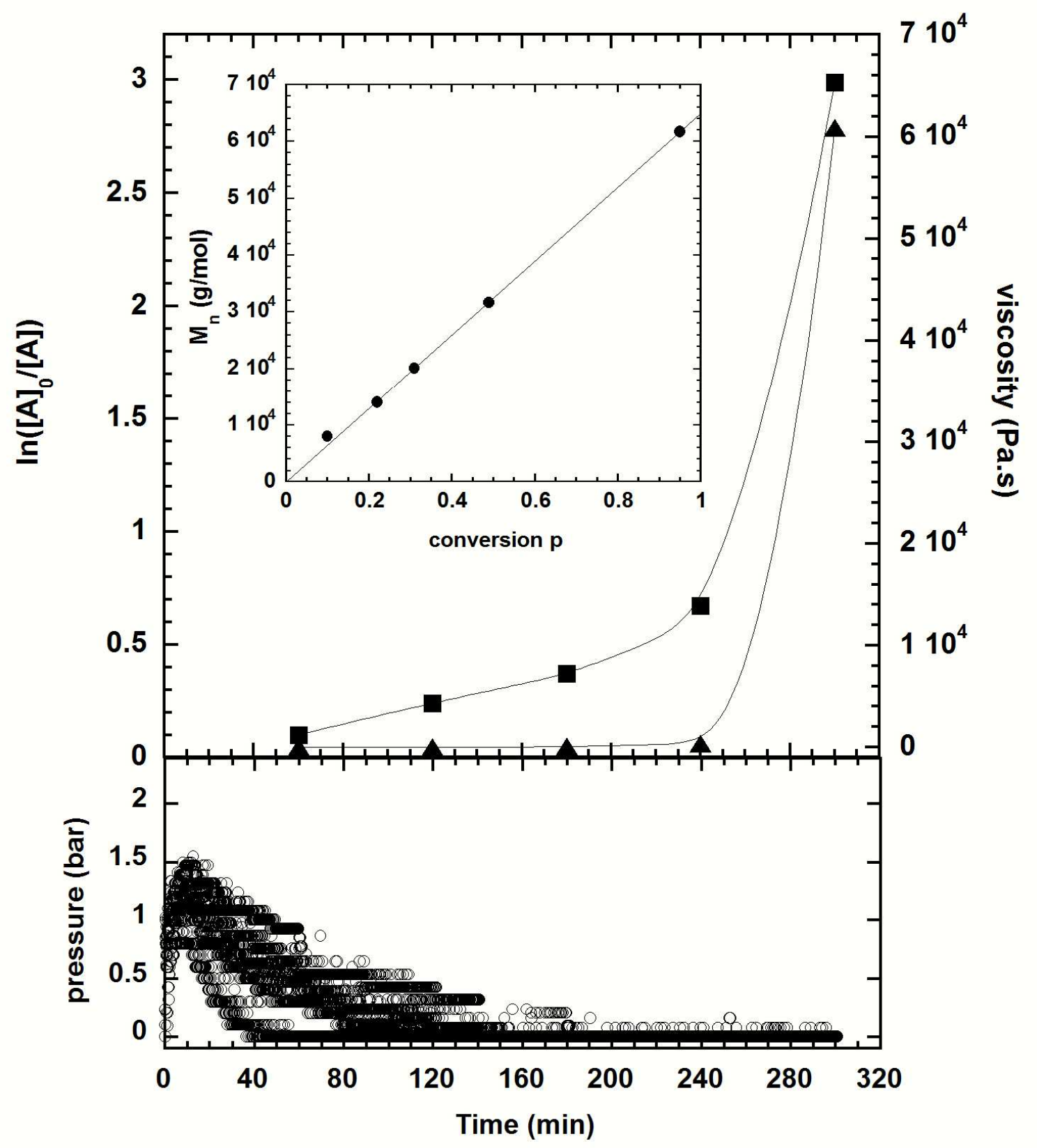


It should be mentioned that a polymer solution at concentrations higher than $\mathrm{c}^{* *}$ enters a socalled concentrated regime in which each segment of the polymer chain lacks sufficient space 42 . The chains thus display a greatly reduced mobility as opposed to their counterparts in dilute $\left(\mathrm{c}<\mathrm{c}^{*}\right)$ or semi-dilute $\left(\mathrm{c}^{*}<\mathrm{c}<\mathrm{c}^{* *}\right)$ solutions, and their viscosity increases very sharply with the concentration and molar mass of the polymer: $\eta \propto c^{x}$ with $x=15 / 4$ in a good solvent, and $\eta \propto \mathrm{M}^{3}$ (where $\mathrm{c}^{*}$ is the overlap concentration). Typically, the volume fraction of the polymer at $c^{* *}$ is between 0.2 and 0.3 . For a polymer of a sufficiently high molar mass, there is a broad range of concentrations between $c^{*}$ and $c^{* *}$. In the present study, the monomer concentration was kept constant at $400 \mathrm{mg} / \mathrm{mL}$ and a polymer concentration higher than $\mathrm{c}^{* *}=200 \mathrm{mg} / \mathrm{mL}$ would correspond to a polymer conversion greater than $\mathrm{p}=0.5$. For all microwave experiments, it appeared that the auto-acceleration of the polymerization rate occurred at a conversion close to 0.5 ( $>0.4$ in figure 5) and with a microwave energy higher than $30 \mathrm{~kJ}$ per gram of reaction mixture.

Thus, for the present work, it can be suggested that the mobility of the long-chain radical species was significantly reduced as the viscosity increased, i.e., the radicals started demonstrating difficulties moving. On the other hand, under the sufficiently high energy of the electric field induced by the pulsed microwave mode, the molecules of water, monomers and counter radicals in the reaction system rotated and oscillated at a high speed before becoming polarized. At this gel point, the reactivity of the reaction system seems to be enhanced, either by increasing the propagation constant of the monomer $\left(\mathrm{k}_{\mathrm{p}}\right)$ and/or by rateenhancing reductions of the persistent radical concentration (e.g., hydroxylamine formation $){ }^{43}$ resulting in the considerable acceleration of the polymerization process without lack of living/controlled polymerization according to the prediction of the persistence radical effect theory, ${ }^{44}$ i.e., the so called Fischer effect. ${ }^{45}$

The explanation that a microwave effect was induced by a mobility contrast between the polymer chain and the dipolar monomer/counter radical from a sufficient microwave energy is not obvious. Probably, the increase of the temperature and the mobility contrast arising from the gelification of the reaction media are concomitant. Future objectives include clarifying the respective parts of the rate-enhancing propagation of monomer, the rateenhancing reductions of the persistent radical concentration and the temperature effect with the use of an internal sensor. 
In addition, Kappe et $a .^{2}$ recently demonstrated that a multiple fiber-optic probe system was an accurate temperature measurement device in both microwave and conventionally heated reactors, and that an efficient stirring/agitation of microwave-heated reactions remains essential. In contrast to an oil bath experiment, even completely homogeneous solutions need to be stirred when single-mode microwave reactors are employed. If efficient stirring/agitation cannot be ensured, temperature gradients may develop as a consequence of inherent field inhomogeneities alongside a single-mode microwave cavity. In the case of homogeneous polymer synthesis in bulk or in solution, the medium can become extremely viscous, thus resembling a pseudo or "physical" gel. If the disruption of the stirring had given rise to inhomogeneities alongside the microwave vial with regard to the temperature, the distribution of the molar masses would have been prevented from reaching very high values. Indeed, the kinetic chain length in radical polymerization is a function of the polymerization rate, which, in turn, depends on the temperature. A too high variation in temperature alongside the microwave vial would have provided a broad distribution in molar masses ${ }^{46}$ which was not observed. Thus, in our experimental device, the temperature along the length of the reactive medium can be supposed to be homogeneous but this feature cannot be extrapolated to bigger volumes.

\section{Conclusions}

The present work has demonstrated that the combined use of microwave irradiation as a heating source and water as a solvent provides a reasonable living/controlled polymerization of acrylamide by NMP. For the reaction, a combination of a conventional hydrosoluble radical initiator (Vazo56) and a $\beta$-phosphonylated nitroxide (SG1) was employed. The microwave enhancement of the polymerization depended on the mode of irradiation. The DYN mode corresponded to a dynamic control of the temperature by use of a high initial microwave power. In this case, no specific microwave effect was observed. For the SPS mode, which is a pulsed power mode, a strong acceleration of the polymerization process (> 50 times) without any loss of the living/controlled polymerization characteristics has been observed. In this case, a relevant efficiency of the re-initiation of the PAM macroinitiator has been demonstrated even after $100 \%$ of conversion of the first block. Moreover, a gelification of the reaction media has been observed with such mode.

The SPS mode seemed to represent a superior means of assuring microwave-assisted nitroxide mediated (co)-polymerization in water to achieve high conversion and low temperatures, i.e. below $90{ }^{\circ} \mathrm{C}$, as measured with external IR sensors. 


\section{Acknowledgments}

The "Conseil Géneral d'Aquitaine" is gratefully acknowledged for the $\mathrm{PhD}$ grant of $\mathrm{J}$. Rigolini and many thanks are expressed to Arkema for providing the SG1 counter radical. We also thank Gérald Clisson for his technical assistance during the SEC experiments and CEM for the loan of the Discover unit.

\section{References}

1. Braunecker, W. A.; Matyjaszewski, K. Progress in Polymer Science 2007, 32, 93-146.

2. Herrero, M. A.; Kremsner, J. M.; Kappe, C. O. Journal of Organic Chemistry 2008, 73, 3647.

3. Kappe, C. O. Angewandte Chemie-International Edition 2004, 43, 6250-6284.

4. Perreux, L.; Loupy, A. Tetrahedron 2001, 57, 9199-9223.

5. Lidstrom, P.; Tierney, J.; Wathey, B.; Westman, J. Tetrahedron 2001, 57, 9225-9283.

6. Tsami, A.; Yang, X. H.; Farrell, T.; Neher, D.; Holder, E. Journal of Polymer Science Part a-Polymer Chemistry 2008, 46, 7794-7808.

7. Lobert, M.; Hoogenboom, R.; Fustin, C. A.; Gohy, J. F.; Schubert, U. S. Journal of Polymer Science Part a-Polymer Chemistry 2008, 46, 5859-5868.

8. Karnati, R.; Ford, W. T. Journal of Polymer Science Part a-Polymer Chemistry 2008, 46, 3813-3819.

9. Xu, Z. S.; Hu, X. X.; Li, X. Q.; Yi, C. F. Journal of Polymer Science Part a-Polymer Chemistry 2008, 46, 481-488.

10. Bardts, M.; Gonsior, N.; Ritter, H. Macromolecular Chemistry and Physics 2008, 209, 2531.

11. Sinnwell, S.; Ritter, H. Australian Journal of Chemistry 2007, 60, 729-743.

12. Hoogenboom, R.; Schubert, U. S. Australian Journal of Chemistry 2009, 62, 181-183.

13. Hoogenboom, R.; Schubert, U. S. Macromolecular Rapid Communications 2007, 28, 368386.

14. Wiesbrock, F.; Hoogenboom, R.; Schubert, U. S. Macromolecular Rapid Communications 2004, 25, 1739-1764.

15. Li, J.; Zhu, X. L.; Zhu, J.; Cheng, Z. P. Radiation Physics and Chemistry 2006, 75, 253258. 
16. Leenen, M.; Wiesbrock, F.; Hoogenboom, R.; Schubert, U. S. E-Polymers 2005.

17. Brown, S. L.; Rayner, C. M.; Graham, S.; Cooper, A.; Rannard, S.; Perrier, S. Chemical Communications 2007, 2145-2147.

18. Zhu, J.; Zhu, X. L.; Zhang, Z. B.; Cheng, Z. P. Journal of Polymer Science Part a-Polymer Chemistry 2006, 44, 6810-6816.

19. Delfosse, S.; Borguet, Y.; Delaude, L.; Demonceau, A. Macromolecular Rapid Communications 2007, 28, 492-503.

20. Zhang, H. Q.; Schubert, U. S. Macromolecular Rapid Communications 2004, 25, 1225 1230.

21. Cheng, Z. P.; Zhu, X. L.; Chen, G. J.; Xu, W. J.; Lu, J. M. Journal of Polymer Science Part a-Polymer Chemistry 2002, 40, 3823-3834.

22. Hou, C.; Guo, Z. L.; Liu, J. S.; Ying, L.; Geng, D. D. Journal of Applied Polymer Science 2007, 104, 1382-1385.

23. Dallinger, D.; Kappe, C. O. Chemical Reviews 2007, 107, 2563-2591.

24. Erdmenger, T.; Becer, C. R.; Hoogenboom, R.; Schubert, U. S. Australian Journal of Chemistry 2009, 62, 58-63.

25. Nuchter, M.; Ondruschka, B.; Bonrath, W.; Gum, A. Green Chemistry 2004, 6, 128-141.

26. Zhang, C.; Liao, L. Q.; Gong, S. Q. S. Green Chemistry 2007, 9, 303-314.

27. Grassl, B.; Clisson, G.; Khoukh, A.; Billon, L. European Polymer Journal 2008, 44, 5058.

28. Ghannam, L.; Garay, H.; Shanahan, M. E. R.; Francois, J.; Billon, L. Chemistry of Materials 2005, 17, 3837-3843.

29. Ghannam, L.; Bacou, M.; Garay, H.; Shanahan, M. E. R.; Francois, J.; Billon, L. Polymer 2004, 45, 7035-7045.

30. Paillet, S.; Grass1, B.; Desbrieres, J. Analytica Chimica Acta 2009, 636, 236-241.

31. Thevenot, C.; Grassl, B.; Bastiat, G.; Binana, W. Colloids and Surfaces aPhysicochemical and Engineering Aspects 2005, 252, 105-111.

32. Nicolay, R.; Marx, L.; Hemery, P.; Matyjaszewski, K. Macromolecules 2007, 40, 60676075 .

33. Phan, T. N. T.; Bertin, D. Macromolecules 2008, 41, 1886-1895.

34. Charleux, B.; Nicolas, J. Polymer 2007, 48, 5813-5833.

35. Lefay, C.; Charleux, B.; Save, M.; Chassenieux, C.; Guerret, O.; Magnet, S. Polymer 2006, 47, 1935-1945.

36. Nicolas, J.; Charleux, B.; Guerret, O.; Magnet, S. Macromolecules 2004, 37, 4453-4463. 
37. Zetterlund, P. B.; Kagawa, Y.; Okubo, M. Chemical Reviews 2008, 108, 3747-3794.

38. Veregin, R. P. N.; Odell, P. G.; Michalak, L. M.; Georges, M. K. Macromolecules 1996, 29, 2746-2754.

39. Souaille, M.; Fischer, H. Macromolecules 2001, 34, 2830-2838.

40. Niki, E. Methods in Enzymology 1990, 186, 100-108.

41. Ananchenko, G. S.; Souaille, M.; Fischer, H.; Le Mercier, C.; Tordo, P. Journal of Polymer Science Part a-Polymer Chemistry 2002, 40, 3264-3283.

42. Teraoka, I. POLYMER SOLUTIONS: An Introduction to Physical Properties; John Wiley \& Sons, 2002.

43. Sciannamea, V.; Jerome, R.; Detrembleur, C. Chemical Reviews 2008, 108, 1104-1126.

44. Souaille, M.; Fischer, H. Macromolecules 2002, 35, 248-261.

45. Fischer, H. Chemical Reviews 2001, 101, 3581-3610.

46. Chauvin, F.; Dufils, P. E.; Gigmes, D.; Guillaneuf, Y.; Marque, S. R. A.; Tordo, P.; Bertin, D. Macromolecules 2006, 39, 5238-5250. 


\section{Graphical Abstract:}

\section{Microwave-Assisted Nitroxide-Mediated Radical Polymerization of Acrylamide in Aqueous Solution}

Julien Rigolini, Bruno Grassl, Laurent Billon, Stephanie Reynaud and Olivier F.X. Donard

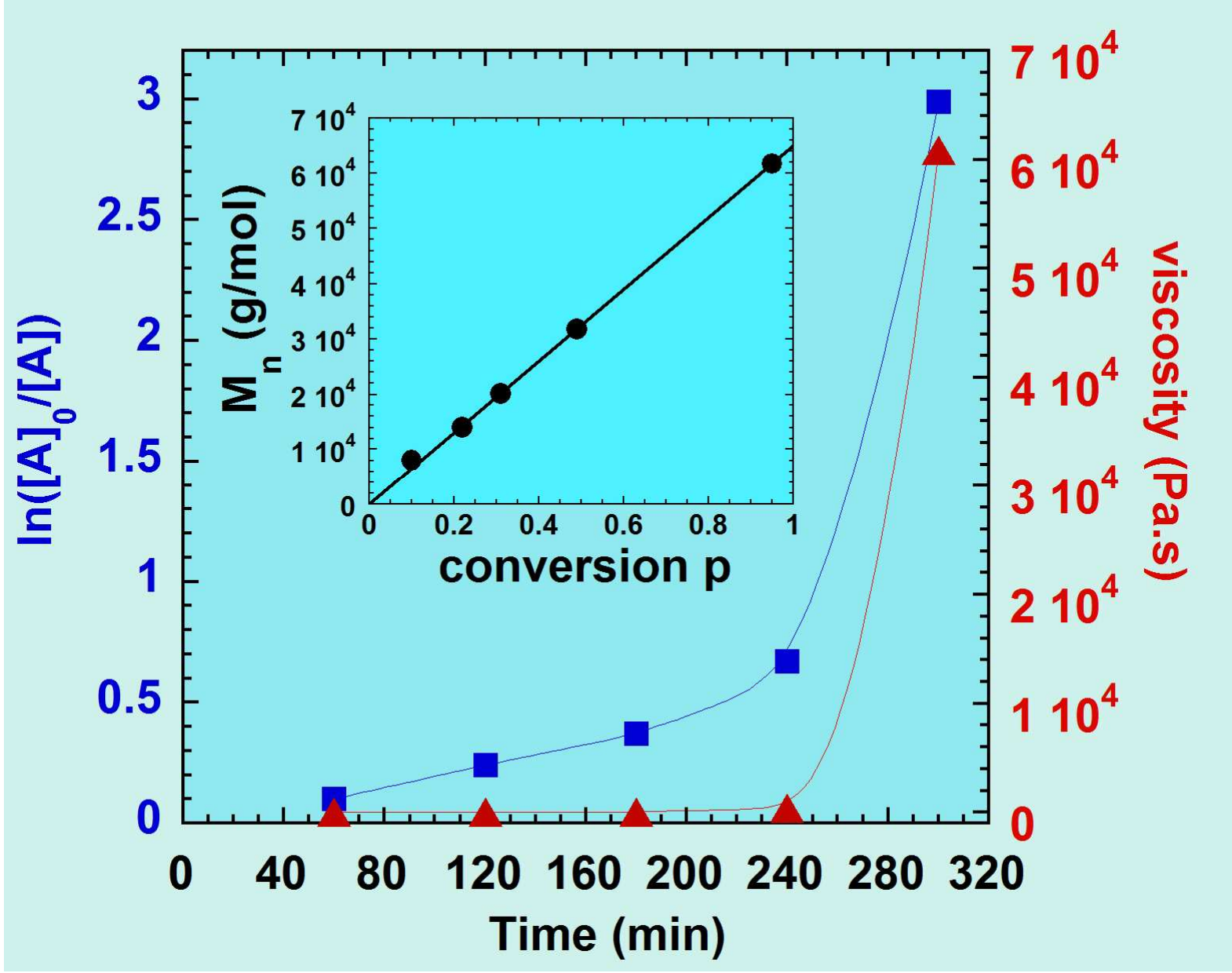

The SPS modes which is a pulsed power mode, represents a superior mean of assuring microwave-assisted nitroxide mediated (co)-polymerization in water at high conversion and low temperatures, i.e., below $90{ }^{\circ} \mathrm{C}$, as measured with external IR sensors. 


\section{Captions to table and figures}

Table 1 : Temperature $(\mathrm{T})$, polymer conversion $(\mathrm{p})$, reaction time $(\mathrm{t})$, number-average molar mass $\left(\mathrm{M}_{\mathrm{n}}\right)$ and polydispersity index (Ip) of the NMP of acrylamide in aqueous solution using conventional heating $(\mathrm{CH})$ and two microwave modes (DYN and SPS): $r=[\mathrm{AM}] / 2[\mathrm{Vazo56}]$ $=500$ for a constant acrylamide (AM) concentration $(40 \mathrm{wt} \%)$ and a constant molar ratio $[\mathrm{SG} 1]_{0} /[\mathrm{Vazo} 56]_{0}=1.8$.

Figure 1: SEC-MALS results for a typical experiment: $\mathrm{CH}$ run, $120^{\circ} \mathrm{C}, \mathrm{t}=20,40$ and $50 \mathrm{~min}$ (see Table 1). a) Chromatograms (top), and b) molar mass distribution (bottom). The three peaks on the left of the top figure correspond to the three peaks in the bottom figure.

Figure 2: Profiles for temperature and power during microwave irradiation in DYN (top) and SPS (bottom) modes.

\section{Figure 3:}

a) Kinetic plots of $\ln \left([\mathrm{AM}]_{0} /[\mathrm{AM}]\right)$ vs. reaction time for the NMP polymerization of acrylamide under conventional heating $(\mathrm{CH})$ and dynamic microwave irradiation (DYN) at various temperatures: $\mathrm{CH}$ (full symbols), $120^{\circ} \mathrm{C}(\boldsymbol{\bullet}), 115^{\circ} \mathrm{C}(\boldsymbol{\square}), 105^{\circ} \mathrm{C}(\bullet), 90^{\circ} \mathrm{C}(\boldsymbol{\nabla})$; DYN (open symbols), $105^{\circ} \mathrm{C}(\diamond), 97^{\circ} \mathrm{C}(\triangle)$ and $90^{\circ} \mathrm{C}(\nabla)$.

b)The dependence of the number-average molar mass $\left(\mathrm{M}_{\mathrm{n}}\right)$ and the polydispersity index (Ip = $\mathrm{Mw} / \mathrm{Mn}$ ), on the monomer conversion, p. Same symbols as in (a). The dashed lines are guides for the eyes.

Figure 4: An Arrhenius plot of the NMP of acrylamide under conventional heating $(\mathrm{CH}$, and dynamic microwave irradiation (DYN, O).

Figure 5: The polymer conversion as a function of the microwave energy during one hour of the NMP of acrylamide under SPS microwave irradiation at various temperatures: $105^{\circ} \mathrm{C}(\boldsymbol{\Delta})$, $90^{\circ} \mathrm{C}(\boldsymbol{\square})$ and $80^{\circ} \mathrm{C}$

Figure 6: Kinetic plots of $\ln \left([\mathrm{AM}]_{0} /[\mathrm{AM}]\right)$ (full symbols) and polymer conversion, $\mathrm{p}$ (open symbols) vs. the reaction time for NMP of acrylamide under pulsed microwave irradiation (SPS) at $90^{\circ} \mathrm{C}$ and varying power: $150 \mathrm{~W}$ (ring) and $190 \mathrm{~W}$ (square).

Figure 7: The number-average molar mass $\left(M_{n}\right)$, and the polydispersity index $(\operatorname{Ip}=M w / M n)$, on the monomer conversion, $\mathrm{p}$, for NMP of acrylamide under pulsed microwave irradiation (SPS) at $105^{\circ} \mathrm{C}(\boldsymbol{\Delta}), 90^{\circ} \mathrm{C}(\boldsymbol{\square})$ and $80^{\circ} \mathrm{C}(\boldsymbol{)})$ for the runs presented in figure 5 and 6 (the dashed lines provide the variation observed in Figure 3 for $\mathrm{CH}$ and DYN mode).

Figure 8: Molar mass distributions, differential for chain extension polymerization: macroinitiator, E1 and E2 (from left to right). 
Figure 9: Kinetic plots of $\ln \left([\mathrm{AM}]_{0} /[\mathrm{AM}]\right)$ vs. reaction time for NMP of acrylamide $(\boldsymbol{\square})$ and the corresponding viscosity of the reaction mixture $(\boldsymbol{A})$, under pulsed microwave irradiation at $80^{\circ} \mathrm{C}$ and $250 \mathrm{~W}$. Inset: the dependence of the number-average molar mass, $\mathrm{Mn}$, on the monomer conversion, p ( ). The lines are guides for the eyes. Below: superposition of measured pressures for each experiment. 